\title{
BOLETÍN CRIMINOLÓGICO
}

La justicia juvenil está acaparando gran parte de la atención de los investigadores de nuestro país en los últimos años. Las recientes reformas legislativas llevadas a cabo unido a la trascendencia que se le otorga a la prevención de la delincuencia juvenil, hace que nos encontremos ante un tema al que cualquier sociedad, preocupada porel aumento de la delincuencia, debe dar máximo protagonismo. El trabajo que se presenta ha sido elaborado por investigadores del Instituto vasco de Criminología y ha sido financiado por el Departamento de Ejecución penal del Gobiemo Vasco. Poco a poco parece estar a dquiriendo mayor importancia la evaluación de normas, programas y medidas, siendo este último el caso del presente estudio.

Palabras clave: menores, delincuencia, justicia juvenil, evaluación, medidas, medio abierto

EVALUACIÓN DE LAS MEDIDAS EN MEDIO ABIERTO DEL PLAN DE JUSTICIA JUVENIL DE LA COMUNIDAD AUTÓNOMA DEL PAÍS VASCO

César San Juan

Estefanía Ocáriz

José Luis de la Cuesta

\section{Introducción}

La evaluación de cualquier política social entraña algunas dificultades debido fundamentalmente a la complejidad de los resultados previsibles y a la multiplicidad de acciones que la conforman. Por otra parte, al tratarse de intervenciones implementadas en el marco de los diferentes sistemas sociales del usuario, puede resultar complicado demostrar que el impacto en la conducta de dichos usuarios, tanto adaptada como desadaptada, es consecuencia directa del éxito o fracaso, respectivamente, de las acciones diseñadas desde la política de justicia juvenil.

En este trabajo se presentan algunos resultados de la Evaluación de la implantación de las Medidas en medio abierto del Plan de Justicia juvenil de la Comunidad autónoma del País Vasco, correspondiente al periodo 2003 - 2004. También se exponen una serie de conclusiones orientadas a incentivar el proceso de mejora de la implementación de dicho Plan, basadas en un análisis transversal de todas las fuentes de análisis.

\section{Fuentes de análisis}

La primera fase de la evaluación consistió en identificar las fuentes de análisis que describimos a continuación:

I. Un análisis DAFO para cuya realización se convocaron varias reuniones con todo el personal implicado en las medidas en medio abierto, entre otros, los $\overline{\text { responsables de ejecución de las }}$ medidas, los técnicos territoriales del Gobierno Vasco, los miembros de los equipos psicosociales y los técnicos del Departamento de Ejecución penal.

II. La base de datos relativos a los expedientes de las sentencias del Servicio de Justicia juvenil del Dpto. de Ejecución penal del Gobierno Vasco.

III. Informes de tasas de resolución publicados por otras comunidades autónomas y,

IV. Los resultados recabados de una serie de entrevistas semiestructuradas a diferentes actores y operadores de la justicia juvenil, a saber, los jueces de menores de todos los juzgados de nuestra comunidad autónoma, los coordinadores de fiscalía de menores, los coordinadores de los equipos psicosociales, los responsables de ejecución de las medidas en medio abierto, así como varios menores que cumplían medida en medio abierto en esos momentos. Dicha entrevista, cuya estructura describimos a continuación, fue realizada de manera personal por una de las investigadoras del equipo:

a) Preguntas relativas a la imposición de la medida: 1) ¿La ley 5/2000 ofrece diversas medidas en medio abierto, cómo valora usted esta cuestión?, ¿considera que cumplen los objetivos para las que están fijadas?. 2) En términos generales, ¿podría identificar los aspectos más positivos del catálogo de medidas en medio abierto y las 


\section{METO DO LO G ÍA}

Para el diseño del proceso de evaluación se ha combinado metodología cualitativa y cuantitativa para lo cual se han utilizado diversas fuentes de análisis. El procesamiento y gestión de la informa ción resultante ha dado lugara una serie de conclusiones de síntesis que se han estructurado en función de tres ejes de evalua ción denominados: Suficiencia, Progreso y Eficacia, cuyos indicadores básicos se identifican a continuación.

El eje de evaluación denominado "Suficiencia del Plan", consta fundamentalmente de dos indicadores básicos: la prevalencia y evolución de medidas en medio abierto, y la colaboración con entidades públicas y privadas (sobre todo, para el caso de las prestaciones en beneficio de la comunidad -PBC's-). El eje de evaluación denominado "Progreso del Plan", consta a su vez de dos indicadores: el criterio de elección de la medida, y la demora en la ejecución de la medida. El eje de evaluación denomina do "Eficacia del Plan", presenta los siguientes indicadores: el grado de cumplimiento de la medida (número de intervenciones y tasa de resolución) y las tasas de reincidencia.

posibilidades de ejecución en el contexto del País Vasco?, ¿y en cuanto a los aspectos negativos o limitaciones más significativas?. 3) Las prestaciones en beneficio de la comunidad son definidas como la medida estrella, ¿qué valor añadido puede concederse a dichas medidas con respecto al resto?. 4) Por el contrario, hay otras medidas que se imponen con muy poca frecuencia (por ejemplo, las tareas socioeducativas), ¿a qué se debe este hecho?. 5) ¿A qué factores atribuye la posible demora entre la comisión del hecho delictivo y la ejecución de la medida?. 6) ¿A qué factores atribuye que en un determinado juzgado puedan imponerse mayor número de medidas en medio abierto que en el resto?. 7) ¿A qué factores atribuye que en un determinado juzgado pueda imponerse con mayor frecuencia una determinada medida en medio abierto?. 8) Uno de los objetivos del plan de justicia juvenil es que el $90 \%$ de las medidas ejecutadas sean en medio abierto. ¿Cómo valora que se fije dicho objetivo a priori?. 9) Criterios establecidos para imponer una medida de régimen abierto. 10) Limitaciones a la hora de imponer una medida en régimen abierto y 11) ¿Considera suficiente el abanico de medidas en régimen abierto ofertadas actualmente?

b) Preguntas relativas a la ejecución de la medida: 1) Dificultades de ejecución de medidas en medio abierto. 2) Beneficios de la ejecución de medidas en medio abierto. 3) Cuál es su experiencia con los chicos y chicas que cumplen una medida de régimen abierto gestionadas por su centro. 4) ¿Existe un perfil de menor que más frecuentemente es objeto de medidas en régimen abierto? 5) ¿Cree que se dispone de todos los medios humanos y materiales necesarios para poder aplicar las medidas en medio abierto más idóneas para cada caso?

c) Guión de preguntas realizadas a los menores cumpliendo medida sobre: 1) $\mathrm{Su}$ valoración personal. 2) El objeto de la medida. 3) La conciencia de reparación. 4) Sus expectativas de futuro y 5) El inicio de carrera delictiva y/o reincidencia

\section{Resultados}

Como fruto de los análisis cualitativos se identificaron una serie de "oportunidades de mejora" dentro del departamento de Justicia juvenil del Gobierno Vasco que podemos sintetizar en los siguientes aspectos:

P Mejorar la coordinación con otros Departamentos y/o instituciones para la creación de nuevos recursos o priorización en los ya existentes de este tipo de menores, y dar accesibilidad prioritaria a través de acuerdos institucionales a recursos para menores (CIP)

P Mejorar la relación con jueces, fiscales y equipos técnicos (información, fluidez, etc) y consensuar con jueces, fiscales y equipos técnicos la utilidad y adecuación de las diferentes medidas

P Agrupar expedientes para poder dar una respuesta global
P Extender a todos los juzgados la asistencia de la entidad pública a juicios de menores con medidas anteriores.

P Lograr una mayor inmediatez en la adopción de medidas desde la denuncia de los hechos.

P Mejorar la colaboración y coordinación con otros recursos e instituciones y darse a conocer mutuamente.

P Aumentar los Centros de día como recurso intermedio entre el medio abierto y el internamiento.

Entre los análisis de carácter cuantitativo nos hemos querido centrar para este trabajo en los siguientes aspectos: la demora, los tipos de hechos que dan lugar a la medida comparándolos, a su vez, con el medio cerrado, y la evolución de las medidas en medio abierto. Para ello, hemos realizado un análisis retrospectivo de una muestra de 240 expedientes de las sentencias registradas en los Juzgados de Menores de San Sebastián, Vitoria y Bilbao desde enero de 2001 a diciembre de 2003. Se procedió a un vaciado de la información de los mismos, identificando una serie de variables clave asociadas al perfil penal y psicosocial de los menores y cuyo análisis puede resultarnos útil para analizar, entre otras cuestiones fundamentales, la demora, la evolución de las medidas y la tasa de reincidencia, apuntadas en un principio como indicadores de Progreso y Eficacia en el diseño de evaluación del Plan de Justicia. 
Figura 1. Evolución de las medidas.

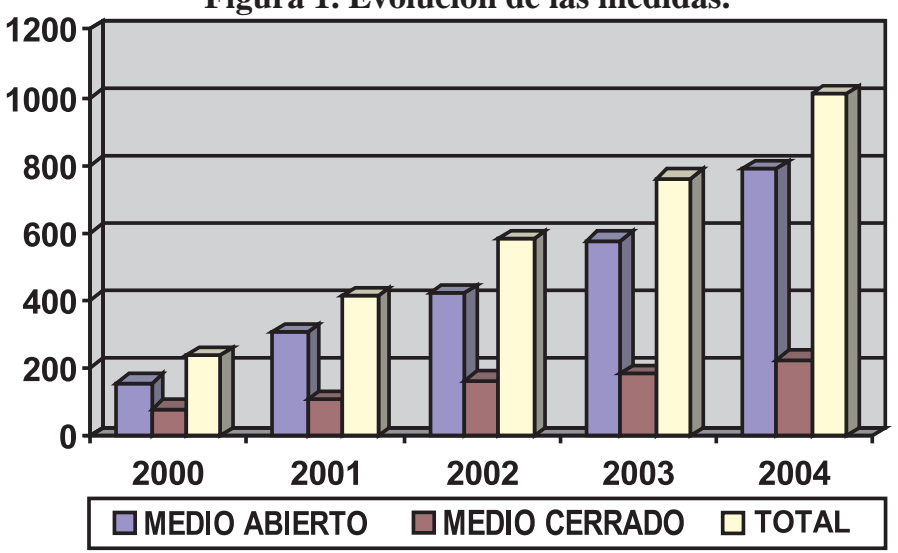

En cuanto a la demora, realizamos dos tipos de análisis. En primer lugar, estudiamos el tiempo que transcurre entre la comisión del hecho por parte del menor y la demanda de ejecución por parte del Juzgado de Menores $\mathrm{y}$, en segundo lugar, el tiempo que transcurre entre la admisión del

hacia el 2004 en torno al $75 \%$. Con todo, si incluimos los procesos de mediación, este porcentaje se vería sensiblemente disminuido.

Por fin, uno de los indicadores quizás más representativos de la eficacia del sistema de justicia es la tasa de reincidencia. Desde Figura 2. Evolución de la tasa de medidas en medio abierto y cerrado en relación al total

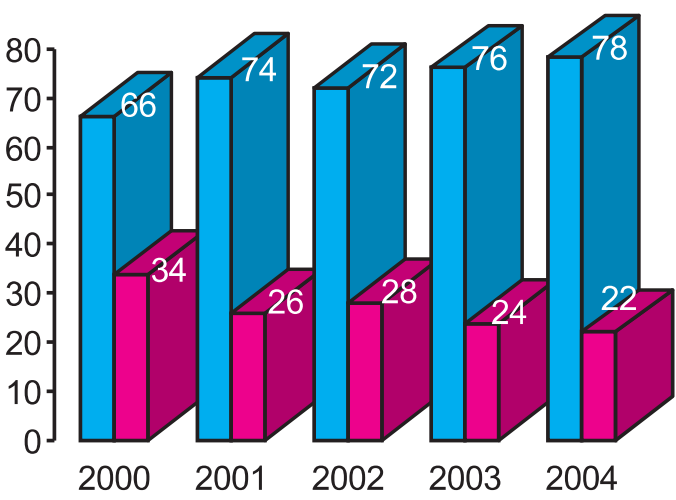

$\square \%$ MEDIO ABIERTO $\square \%$ MEDIO CERRADO

este punto de vista, también planteamos una propuesta de modelo explicativo de la reincidencia que pueda resultar útil en el diseño de políticas de prevención primaria.

Tal y como se aprecia en la figura 3, el porcentaje de menores reincidentes cuya primera medida fue en medio abierto es ligeramente inferior al porcentaje de menores

expediente por parte del Gobierno Vasco y la ejecución efectiva de la medida por parte del menor. En ambos casos, la demora era muy importante, con una media algo superior a siete meses.

Por otra parte, el número de medidas impuestas experimenta un crecimiento anual sostenido. Con la misma tendencia aumenta el número de medidas en medio abierto (Figura 1). Sin embargo, para valorar su incremento relativo debe compararse con el número total de medidas (Figura 2). Así, el porcentaje de medidas en medio abierto experimenta un despunte después de la entrada en vigor de la LO 5/2000, para estabilizarse fue en medio cerrado $\left(\mathrm{X}^{2}=3,54\right.$ $\mathrm{gl}=1 ; \mathrm{P}<0,002)$. Esta tendencia de mayor eficacia de resultados en medio abierto con respecto al medio cerrado puede estar condicionado por el hecho de que las intervenciones en medio cerrado se aplican a delitos más graves, $(r=.27)$ pero lo cierto es que la variable gravedad del primer delito no se correlaciona con el número de delitos cometidos posteriormente. Tras estos análisis exploratorios, podemos hipotetizar un primer modelo de predicción de la reincidencia.

Figura 3: Comparación de porcentaje de reincidentes en función de la primera intervención

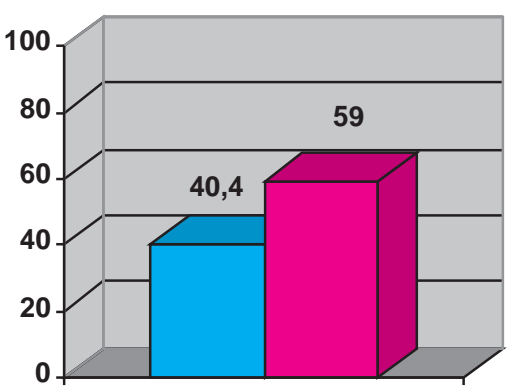

MEDIO ABIERTO $\square$ MEDIO CERRADO

(*) Elaboración propia. $\odot$ IVAC / KREI. 2006

Las dos variables que mejor explican la reincidencia $\left(\mathrm{R}^{2}=0.20\right)$ (Figura 4) son, por un lado, la primera medida que recibe el menor, en el sentido de que el medio cerrado hace más probable que el menor vuelva a delinquir, no influyendo la gravedad del primer delito. Por otra parte, el fracaso escolar se convierte en el mejor predictor de la reincidencia. Cabe añadir que el control paterno, incluido en una hipótesis inicial, no explica de forma significativa la reincidencia. Se confirma la idoneidad de la adaptación a la escolarización como escenario privilegiado de prevención de la delincuencia.

\section{REINCIDENCIA}

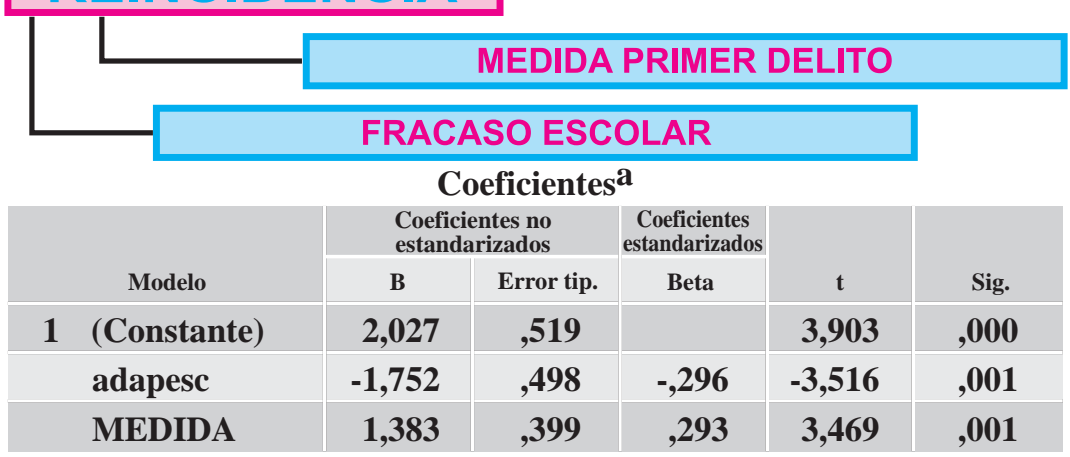

Figura 3: Modelo explicativo de la reincidencia (*) Elaboración propia. (C) IVAC / KREI. 2006 
Otro indicador que puede resultar relevante es la tasa de resolución, que solo adquiere un cierto significado si la comparamos con los resultados obtenidos por otros servicios de justicia de nuestro entorno. Así, se puede comprobar (Figuras $5 y$ 6) que en relación a otras comunidades o provincias como, por ejemplo, Madrid, Orense o Asturias, la CAPV tiene una mayor tasa de resolución de expedientes (2003-2004).
Se ha de destacar así mismo que en cuanto a la medida de PBC se aprecia también el interés especial de la CAPV por esta medida, entre todas las de medio abierto.

Figuras 5 y 6: Comparación de tasa de resolución y PBC con otras CC.AA

TASA DE RESOLUCIÓN

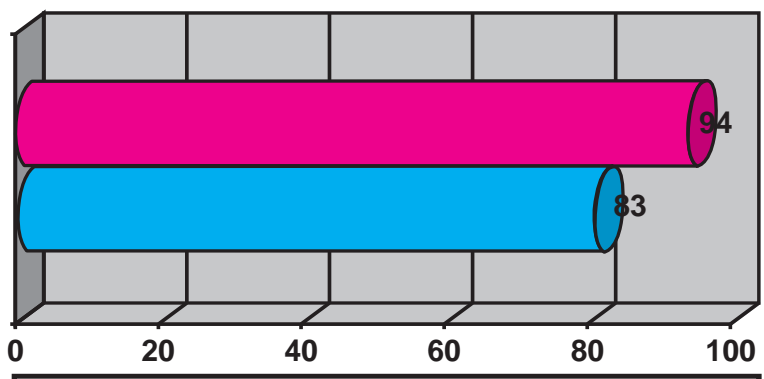

C.A.P.V. $\square$ MADRID, ASTURIAS Y ORENSE
TASA DE MEDIDAS PBC/MEDIO ABIERTO

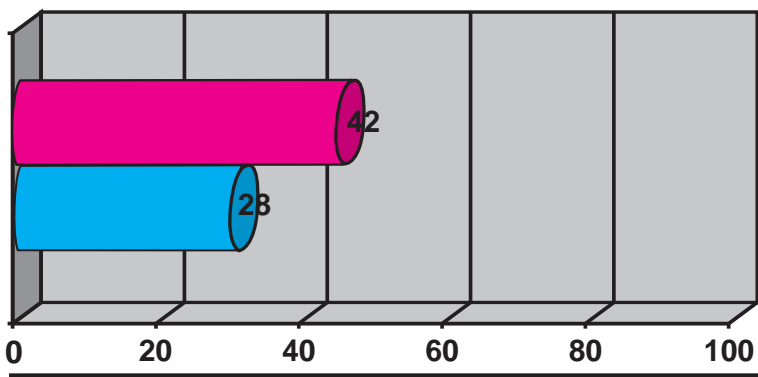

C.A.P.V. $\square$ MADRID, ASTURIAS Y ORENSE

(*) Elaboración propia. (C) IVAC / KREI. 2006

\section{O NCLUSIO NES}

Retomando la introducción de esta evaluación, pasamos a explicar brevemente los resultados principales de cada eje de evaluación, tomando como base los indicadores ya detallados. a) Suficiencia

En este sentido, en cuanto a la prevalencia y evolución de las medidas en medio abierto, diremos en términos absolutos que las medidas en medio abierto experimentan un crecimiento sostenido desde el año 2001.

Con respecto a la colaboración con entidades públicasy privadas, todos los agentes implicados en el Sistema de justicia juvenil perciben la necesidad de incrementar la colaboración con otras entidades fundamentalmente para aumentarla eficacia de las PBC. Así mismo, no puede descartarse la posibilidad de que las entidades colaboradoras que programan las PBC y otras, constituyan un servic io financiado porla Consejería. Solo de esta forma se pueden exigir unos criterios de calidad.

\section{b) Progreso}

Sobre los criterios de elección de lasmedidas, existe una cierta discrepancia sobre la conveniencia de fijar a priori una tasa de medidas en medio abierto. Para jueces, fiscales y equipos téc nicos puede contemplarse como una conveniencia ante la diversidad de opciones, pero no como un objetivo. L que prima como criterio es el "interés superior del menor". Por otra parte, las PBC no son idóneas "por definición". Para que el menor pueda encontrarles un sentido, es preciso que el programa guarde una coherencia reparadora con el hecho cometido. Y esto no siempre se consigue.

En cuanto a la demora en la ejecución de las medidas, los análisis parecen indicar una tasa de demora significativamente alta. Es aconsejable adoptar medidas para paliar esta limitación ya que incide directamente en la eficacia de los programas educativos. En todo caso nuestros análisis no mostraron diferencias significativas en la reincidencia en función de la tasa de demora.

\section{c) Eficacia:}

Respecto al grado de cumplimiento y tasas de resolución, el grado de cumplimiento de las intervenc iones muestra unas tasas algo superiores a los datos disponibles para el año 2004 de Madrid, Principado de Asturias y Orense.

En cuanto al grado de involucración del menor, como principio general, podríamos decir que en aquellos casos en los que el menor delinque "accidentalmente", es decir, no forma parte de su patrón de conducta habitual y goza de un soporte familiarestructurado, responde muy adecuadamente a la medida. La situa ción se complica exponencialmente en aquellos casos en los que el menor tiene interioriza do un estilo delictivo y no cuenta con un soporte social sólido de referencia. En estos casos, tanto los equipos psic osociales, como los propios menores, destacan la importancia fundamental del educador en detrimento de las caracteństicas de la medida. Es decir, parece más eficaz un buen educador que una buena medida.

Por último, en cuanto a la tasa de reincidencia, según nuestros a nálisis, el fracaso escolar es la variable que mejor predice la reincidencia. A continuación, la aplicación de una medida en medio cerrado en el primer delito, independientemente de su gravedad, también predice significativamente la reincidencia. Asumiendo la reincidencia como un indicador de eficacia del sistema, parece avalarse la hipótesis de la conveniencia del medio abierto como respuesta judicial y, por otra parte, se destaca la importancia del medio escolar como entomo privilegiado de prevención de la delincuencia. 